\title{
Engineering Sustainability: Referees 2017
}

The following is a list of referees who have reviewed papers for Engineering Sustainability between 1 December 2016 and 30 November 2017. The Institution of Civil Engineers is very grateful for their assistance.

We are continually looking for suitable reviewers for papers submitted to Engineering Sustainability. Papers published in the Proceedings of the ICE must be submitted to at least two independent referees to judge accuracy, style, impact, importance and interest.

If you are interested in reviewing articles on any topic related to engineering sustainability, please submit your name, qualifications or $\mathrm{CV}$, and areas of expertise. We are in need of individuals who will agree to review papers in a timely fashion (within 3 to 4 weeks of receipt) and provide confidential feedback to the Editorial Advisory Panel concerning the quality of the paper and any suggested revisions that would be appropriate.

If you are such a person, please contact Rebecca Rivers (tel.: +44 (0)207 665 2241; e-mail: rebecca.rivers@ice.org.uk) for more information on the referee process.

$\begin{array}{ll}\text { M. Ahmed } & \text { A. Dannatt } \\ \text { L. Anstead } & \text { T. Downs } \\ \text { J. Atkinson } & \text { G. Everett } \\ \text { H. M. Aziz } & \text { L. Fedele } \\ \text { K. Beven } & \text { S. Fisher } \\ \text { J. Brennan } & \text { J. Fong } \\ \text { T. Brezina } & \text { E. Friedrich } \\ \text { C. Broadbent } & \text { J. Glass } \\ \text { M. Browning } & \text { R. Grzybek } \\ \text { V. Bunster } & \text { P. Guthrie } \\ \text { A. Cambridge } & \text { L. Hartley } \\ \text { R. Campen } & \text { T. Haupt } \\ \text { R. Chowdhury } & \text { A. Henriques } \\ \text { E. Coakley } & \text { A. Hojjati } \\ \text { A.-S. Colas } & \text { A. Hollberg } \\ \text { A. Constantinou } & \text { G. Hu } \\ \text { M. Cook } & \text { E. Huggins } \\ \text { S. Dadson } & \text { C. Jayasinghe }\end{array}$

J. Jeffers

S. Kandasami

F. Karim

E. Karol

R. Kellagher

B. Lin

Y. Lu

K. H. Mo

A. Moncaster

M. Montgomery

S. Motamedi

B. Nastasi

L. Nguyen

M. Norbury

D. Ouahrani

A. Owen

F. Paige

W. Pan
D. Porter
A. Price
M. Radhakrishnan
A. Raut
M. Reiner
S. Sadr
D. Savic
J. Simm
A. Smith
S. Tahir
O. Tarrant
M. Tight
M. Valipour
W. Wallace
B. Weil
L. Zhang
J. Zuo 